\title{
CMR findings in patients with hypertrophic cardiomyopathy and
} atrial fibrillation

\author{
Theano Papavassiliu* ${ }^{* 1}$, Tjeerd Germans ${ }^{\dagger 2}$, Stephan Flüchter ${ }^{1}$, \\ Christina Doesch ${ }^{1}$, Anton Suriyakamar ${ }^{1}$, Dariusch Haghi ${ }^{1}$, Tim Süselbeck ${ }^{1}$, \\ Christian Wolpert ${ }^{1}$, Dietmar Dinter ${ }^{2}$, Stefan O Schoenberg ${ }^{3}$, Albert C van \\ Rossum $^{2}$ and Martin Borggrefe ${ }^{1}$
}

\begin{abstract}
Address: ${ }^{1} 1$ st Department of Medicine-Cardiology, University Hospital of Mannheim, Mannheim, Germany, ${ }^{2}$ Department of Cardiology, VU University Medical Center, Amsterdam, the Netherlands and ${ }^{3}$ Department of Radiology, University Hospital of Mannheim, Germany

Email: Theano Papavassiliu* - theano.papavassiliu@umm.de; Tjeerd Germans - T.Germans@vumc.nl;

Stephan Flüchter - stephan.fluechter@umm.de; Christina Doesch - christina.doesch@umm.de; Anton Suriyakamar - asuriyak@gmx.net; Dariusch Haghi - dariusch.haghi@umm.de; Tim Süselbeck - tim.sueselbeck@umm.de; Christian Wolpert - christian.wolpert@umm.de; Dietmar Dinter - dietmar.dinter@umm.de; Stefan O Schoenberg - stefan.schoenberg@umm.de; Albert C van Rossum - ac.vrossum@vumc.nl; Martin Borggrefe - martin.borggrefe@umm.de
\end{abstract}

* Corresponding author †Equal contributors

\section{Published: 9 September 2009}

Journal of Cardiovascular Magnetic Resonance 2009, I I:34 doi:10.1 186/1532-429X-1 I-34

This article is available from: http://www.jcmr-online.com/content/II/I/34

(C) 2009 Papavassiliu et al; licensee BioMed Central Ltd.

This is an Open Access article distributed under the terms of the Creative Commons Attribution License (http://creativecommons.org/licenses/by/2.0), which permits unrestricted use, distribution, and reproduction in any medium, provided the original work is properly cited.
Received: 30 July 2009

Accepted: 9 September 2009

\begin{abstract}
Objectives: We sought to evaluate the relation between atrial fibrillation (AF) and the extent of myocardial scarring together with left ventricular (LV) and atrial parameters assessed by late gadolinium-enhancement (LGE) cardiovascular magnetic resonance $(C M R)$ in patients with hypertrophic cardiomyopathy $(\mathrm{HCM})$.
\end{abstract}

Background: AF is the most common arrhythmia in HCM. Myocardial scarring is also identified frequently in HCM. However, the impact of myocardial scarring assessed by LGE CMR on the presence of AF has not been evaluated yet.

Methods: 87 HCM patients underwent LGE CMR, echocardiography and regular ECG recordings. LV function, volumes, myocardial thickness, left atrial (LA) volume and the extent of LGE, were assessed using CMR and correlated to AF. Additionally, the presence of diastolic dysfunction and mitral regurgitation were obtained by echocardiography and also correlated to AF.

Results: Episodes of AF were documented in 37 patients (42\%). Indexed LV volumes and mass were comparable between HCM patients with and without AF. However, indexed LA volume was significantly higher in HCM patients with AF than in HCM patients without AF $\left(68 \pm 24 \mathrm{ml} \cdot \mathrm{m}^{-2}\right.$ versus $46 \pm 18 \mathrm{ml} \cdot \mathrm{m}^{-2}, \mathrm{p}=0.0002$, respectively). The mean extent of LGE was higher in HCM patients with AF than those without $A F(12.4 \pm 14.5 \%$ versus $6.0 \pm 8.6 \%, p=0.02)$. When adjusting for age, gender and $L V$ mass, $L G E$ and indexed LA volume significantly correlated to $A F(r=0.34, p=0.02$ and $r=0.42, p<0.001$ respectively). By echocardiographic examination, LV diastolic dysfunction was evident in 35 (40\%) patients. Mitral regurgitation greater than II was observed in 12 patients (14\%). Multivariate analysis demonstrated that LA volume and presence of diastolic dysfunction were the only independent determinant of $A F$ in $H C M$ patients $(p=0.006, p=0.01$ respectively). Receiver operating characteristic curve analysis indicated good predictive performance of LA volume and LGE (AUC $=0.74$ and 0.64 respectively) with respect to AF.

Conclusion: HCM patients with AF display significantly more LGE than HCM patients without AF. However, the extent of LGE is inferior to the LA size for predicting AF prevalence. LA dilation is the strongest determinant of $A F$ in $H C M$ patients, and is related to the extent of $L G E$ in the $L V$, irrespective of $L V$ mass. 


\section{Introduction}

Hypertrophic cardiomyopathy (HCM) is a complex and relatively common form of genetic heart disease and the most frequent cause of sudden death in the young [1]. HCM is macroscopically characterized by -often asymmetrical - left ventricular (LV) hypertrophy in the absence of any systemic or cardiac disease likely to cause this hypertrophy. Histologically, myocyte disarray, scarring and microvascular dysfunction are the hallmarks of HCM.

Atrial fibrillation (AF) is the most common arrhythmia in HCM [2-5]. In general, AF is associated with unfavourable prognosis secondary to an increased risk of heart failurerelated mortality, thrombo-embolism and severe functional impairment [6-10]. In HCM patients, increased left atrial (LA) size, advanced age and congestive heart failure symptoms have been shown to be independent predictors of the occurrence of AF [11]. However, the effect of myocardial scarring on the presence of AF has not been evaluated yet.

Cardiovascular magnetic resonance (CMR) has a high spatial resolution, is considered the gold standard for in vivo determination of left ventricular (LV) mass and volumes, and enables precise quantification of wall thickness and dimensions [12,13]. Additionally, late gadoliniumenhanced (LGE) CMR allows visualization of myocardial scarring in HCM patients $[14,15]$.

Given the association between the extent of LGE with progressive LV dilation, ventricular arrhythmias and markers of sudden death in HCM patients we hypothesized that the extent of LGE might also be associated with the presence of AF [16-18]. Thus, we used CMR and echocardiography to evaluate whether the extent of LGE, LV and LA size, diastolic dysfunction and mitral regurgitation were associated with AF in HCM patients.

\section{Methods}

\section{Study population}

In total, 102 consecutive HCM patients referred for CMR were selected between February 2003 and December 2006 at 2 referral centers: $1^{\text {st }}$ Department of Medicine, University Hospital of Mannheim, Germany and the Department of Cardiology, VU University Medical Center, Amsterdam, the Netherlands. All HCM patients were diagnosed based on conventional criteria; left ventricular hypertrophy $\geq 15$ $\mathrm{mm}$ on two-dimensional echocardiography in the absence of another disease that could account for the hypertrophy [19]. The work-up at intial diagnosis included electrocardiogram (ECG), echocardiography, coronary angiography, left ventriculography, 24-h Holter ECG and CMR imaging. Every 6 months the majority of patients underwent follow up visits including ECG, echocardiography and 24-h Holter ECG. Of 102 HCM patients, 15 patients were excluded due to concomitant coronary artery disease $(n=4)$, previous myocardial infarction ( $\mathrm{n}=3)$ or implantation of a pacemaker or defibrillator $(\mathrm{n}=8)$, yielding a total of 87 patients included finally in this study (54 males and 33 females; mean age $58 \pm 13$ years). This study was approved by the institutional ethics committees of both participating medical centers and informed consent was obtained from all subjects.

\section{CMR acquisition}

All studies were performed using a 1.5 Tesla whole body imaging system (Magnetom Sonata, Siemens Medical Systems, Erlangen, Germany). A dedicated four-element, phased-array body coil was used. Images were acquired during breath-holds in mild expiration. Scout images (coronal, sagittal and axial planes) were obtained for planning of the final double-oblique long-axis and shortaxis views. To evaluate functional parameters, ECG-gated cine images were then acquired using a balanced segmented steady state free precession (trueFISP) sequence. Typical scan parameters were: $5 \mathrm{~mm}$ slice thickness with 5 $\mathrm{mm}$ interslice gap, temporal resolution $35 \mathrm{~ms}$, repetition time $3.2 \mathrm{~ms}$, echo time $1.2 \mathrm{~ms}$, flip angle 60 degrees, and typical in-plane spatial resolution $1.4 \times 1.8 \mathrm{~mm}^{2}$. After obtaining standard 4, 3 and 2 chamber long axis cines, a stack of 9 to 12 short-axis slices was used for full coverage of the left and right ventricle. The LGE images were obtained 10-15 min after intravenous administration of $0.2 \mathrm{mmol} \cdot \mathrm{kg}^{-1}$ gadolinium-DTPA (Magnevist, Schering AG, Berlin, Germany), using an inversion recovery turbo Fast Low Angle Shot (FLASH) sequence with $6 \mathrm{~mm}$ slice thickness at the same position as the long- and short-axis cines in end diastole [20]. The inversion time was adjusted per patient to optimally null signal from normal myocardium typically between 250 and $300 \mathrm{~ms}$. Total acquisition time averaged $40 \mathrm{~min}$.

\section{Left ventricular and left atrial image analysis using CMR}

LV end diastolic volumes, LV end-systolic volumes, LV stroke volume, LV ejection fraction and LV myocardial mass were assessed off-line from the serial short-axis true FISP cine loops using dedicated commercially available software (ARGUS, Siemens, Erlangen, Germany). In addition to volumetric measurements, one-dimensional measurements of LV end diastolic dimensions (LVEDD), posterior wall thickness (PWT) and maximum interventricular septum wall thickness (SWT) were measured from end diastolic short-axis views. LA volumes were measured at end systole by the biplane area-length method on 4 and 2 chamber long axis views [21].

The extent of LGE was quantified using semi-automatic commercially available software (MASS, Medis, Leiden, the Netherlands) defining a signal intensity over 2 stand- 
ard deviations above the mean signal intensity of a region of interest drawn in remote myocardium in the same slice as where the LGE was present [14]. The extent of LGE was then expressed as a percentage of the total LV mass to enable comparison of LGE burden between patients with different LV mass. LV and LA dimension and extent of LGE were measured by 2 observers blinded to all clinical patient details (TP; TG).

\section{Echocardiography}

Standard echocardiographic images were obtained using a Vivid 7 machine (GE Ultrasound, Horton, Norway) with a $2.5 \mathrm{MHz}$ phased array transducer at end-expiratory apnea.

Peak velocity of early (E) and late (A) wave of transmitral flow and E-wave deceleration time (DT) were measured from the pulsed-wave Doppler (PWD) obtained at the tip of mitral leaflets. Tissue Doppler imaging (TDI) was used to obtain early diastolic LV myocardial velocities (E') in the apical 4- and 2-chamber views with a $2 \mathrm{~mm}$ sample volume placed at the lateral, septal, anterior, and inferior mitral annulus. The average of the four annular sites ( $E_{\text {glo- }}^{\prime}$ bal) was used. All echocardiographic measurements were averaged over three consecutive cardiac cycles, measured by a single investigator blinded to all other variables. LV diastolic dysfunction was defined as follows: [1] PWD criteria: $\mathrm{E} / \mathrm{A}$ ratio $<1$ if age $<55$ or $<0.8$ if age $\geq 55$, and/or DT $>240 \mathrm{~ms}$; [2] TDI criteria: $\mathrm{E}_{\text {global }}^{\prime} \leq 12.9 \mathrm{~cm} / \mathrm{s}$ if age $<40$; $\mathrm{E}_{\text {global }}^{\prime} \leq 10.2 \mathrm{~cm} / \mathrm{s}$ if age 40-59; and $\mathrm{E}_{\text {global }}^{\prime} \leq 7.2 \mathrm{~cm} / \mathrm{s}$ if age $\geq 60$. Color Doppler flow imaging was used for semiquantitative assessment of mitral regurgitation.

\section{Atrial Fibrillation}

AF was documented based on ECG recordings obtained when patients presented with acute onset of symptoms or during follow up visits including 24 hour ambulatory Holter ECG monitoring. AF was classified into paroxysmal and persistent. AF was considered paroxysmal when self-terminating and persistent when sustained over 7 days. In our study only patients were included with episodes of atrial fibrillation of at least 1 hour.

\section{Statistical Analysis}

All data are presented as a mean \pm standard deviation. For comparing LV ejection fraction, LV end-systolic volume, LA volume, LV mass, and percentage of LGE between HCM patients with and without $\mathrm{AF}$, an unpaired, 2-tailed student's t-test was used. A Chi-square test was used to evaluate if the presence of LGE and symptoms were different between HCM patients with and without AF. To assess differences in LV and LA dimensions and extent of LGE between asymptomatic (NYHA I) and symptomatic HCM patients (NYHA II-IV), an unpaired, 2-tailed student's ttest was also used. Pearson's correlation was used to correlate extent of LGE with LA volume and LV mass. Partial correlation was used to correct the correlation between the extent of LGE and LA volume with presence of AF for age, gender and LV mass. Multivariate analysis was performed with logistic regression analysis using block entry of the following variables: LA volume, extent of LGE, LV mass, presence of mitral regurgitation, presence of diastolic dysfunction, age and gender to evaluate if these variables were independent predictors of $\mathrm{AF}$, provided to have a $\mathrm{p}<0.10$ in univariate analysis. Area-under-curve analysis was performed to evaluate if LA volume, extent of LGE and LV mass would increase the likelihood of having AF in HCM patients. Additionally, receiver-operator-curve analysis was used to determine the optional cut-off value of LA volume and extent of LGE to discriminate patients at increased risk of AF from patients without. All results were considered statistically significant when $\mathrm{p}<0.05$. Analyses were performed with Statistical Package for Social Sciences (SPSS for windows 14.0, Chicago, IL, USA).

\section{Results}

In 87 of included HCM patients, AF was documented in 37 HCM patients (43\%) at time of initial diagnosis or during follow-up. Baseline characteristics are presented in table 1 . AF was classified as paroxysmal in 27 patients $(31 \%)$ and persistent in 10 patients $(12 \%)$. According to the NYHA classification system, 41 (47\%) were asymptomatic (NYHA functional class I) and the other 46 (53\%) were symptomatic (NYHA functional classes II-IV). By echocardiographic examination, LV diastolic dysfunction was evident in $35(40 \%)$ patients. Mitral regurgitation greater than II was observed in 12 patients (14\%). A total of 45 patients (52\%) were taking beta-blockers, $16(18 \%)$ calcium-channel blockers, 9 (10\%) amiodarone, 3 (3\%) digitalis and $1(1 \%)$ flecainide. A similar proportion of patients in the $\mathrm{AF}$ and non $\mathrm{AF}$ subgroups were taking cardioactive medications.

Age, LV ejection fraction, LV end systolic volume, and LV mass were all comparable between HCM patients with and without AF. However, LV end systolic volume tended to be larger and LV ejection fraction tended to be lower in HCM patients with AF, see table 2. LA volume was significantly larger in $\mathrm{HCM}$ patients with $\mathrm{AF}$ than in patients without $\mathrm{AF}(\mathrm{p}=0.0002)$. In symptomatic HCM patients, LV end systolic volume $(\mathrm{p}=0.04)$, LA volume $(\mathrm{p}=0.03)$ and extent of LGE ( $p=0.03$ ) were significantly larger compared to asymptomatic HCM patients, see table 3 . AF occurred in $41 \%$ of asymptomatic HCM patients and in $43 \%$ of symptomatic HCM patients, which was not significant. In symptomatic patients the percentage of persistent AF tended to be higher compared to asymptomatic patients $(17 \%$ versus $5 \%, \mathrm{p}=0.13)$.

In general, in our collective LGE occurred in a patchy distribution with multiple foci within hypertrophied regions 
Table I: Patient Demographics and Baseline Characteristics.

\begin{tabular}{|c|c|c|c|}
\hline & All Patients $(n=87)$ & Patients with AF $(n=37)$ & Patients without AF $(n=50)$ \\
\hline Age (years) & $58 \pm 13$ & $59 \pm 15$ & $56 \pm 11$ \\
\hline Male gender & $54(61 \%)$ & $22(59 \%)$ & $32(64 \%)$ \\
\hline Hypertension & $8(9 \%)$ & $3(8 \%)$ & $5(10 \%)$ \\
\hline Diabetes mellitus & $6(7 \%)$ & $3(8 \%)$ & $3(6 \%)$ \\
\hline \multicolumn{4}{|l|}{ NYHA functional class } \\
\hline 1 & $41(47 \%)$ & $17(46 \%)$ & $24(48 \%)$ \\
\hline ॥ & $38(44 \%)$ & $16(43 \%)$ & $22(44 \%)$ \\
\hline III/IV & $8(9 \%)$ & $4(10 \%)$ & $4(8 \%)$ \\
\hline Family history of HCM or sudden death & $20(23 \%)$ & $9(24 \%)$ & $\mathrm{II}(22 \%)$ \\
\hline Syncope & $6(7 \%)$ & $4(10 \%)$ & $2(4 \%)$ \\
\hline Atrial fibrillation & $37(43 \%)$ & & \\
\hline None & $50(57 \%)$ & 0 & $50(100 \%)$ \\
\hline Paroxysmal & $27(31 \%)$ & $27(73 \%)$ & 0 \\
\hline Persistent & $10(12 \%)$ & $10(27 \%)$ & 0 \\
\hline \multicolumn{4}{|l|}{ Echocardiographic data } \\
\hline LV diastolic dysfunction, $\mathrm{n}(\%)$ & $35(40 \%)$ & $22(59 \%)$ & $13(26 \%)$ \\
\hline \multicolumn{4}{|l|}{ Mitral regurgitation } \\
\hline I & $27(31 \%)$ & $15(40 \%)$ & $12(24 \%)$ \\
\hline$\geq 2$ & $12(14 \%)$ & 7 (I8\%) & $5(10 \%)$ \\
\hline \multicolumn{4}{|l|}{ Medications, $n(\%)$} \\
\hline Beta-blocker & $45(52 \%)$ & $20(55 \%)$ & $25(49 \%)$ \\
\hline Calcium-channel blocker & $16(18 \%)$ & $7(18 \%)$ & $9(18 \%)$ \\
\hline Amiodarone & $9(10 \%)$ & $5(13 \%)$ & $4(8 \%)$ \\
\hline
\end{tabular}

Data are presented as the mean value \pm SD or number (\%) of subjects. NYHA $=$ New York Heart Association; HCM = hypertrophic cardiomyopathy; LV = left ventricular

of the intervenricular septum, the anterior and posterior walls. In 14 patients LGE was limited at the RV insertion points $(16 / 59=27 \%)$. In two patients LGE was also present in the lateral wall $(2 / 59=3 \%)$. In 13 patients LGE $(13 / 59=22 \%)$ was diffuse trans-septal and RV septal. In these patients, 10 had AF. However, the small sample size precludes any firm conclusions.

The mean extent of LGE was higher in HCM patients with AF compared to those without $(12.4 \pm 14.5 \%$ versus $6.0 \pm$ $8.6 \%, \mathrm{p}=0.02)$. However, the prevalence of AF was comparable between HCM patients with LGE to those without
LGE $(27 / 59,46 \%$ versus $10 / 28,35 \%, \mathrm{p}=0.5)$. When adjusting for age, gender and LV mass, both the extent of LGE and LA volume moderately correlated to $\mathrm{AF}(\mathrm{r}=0.34$, $\mathrm{p}=0.02$ and $0.42, \mathrm{p}<0.001$ respectively). Interestingly, LA volume moderately correlated to the extent of LGE ( $\mathrm{r}=$ $0.31, \mathrm{p}=0.035)$, irrespective of LV mass, which did not correlate with LA volume $(\mathrm{r}=0.08, \mathrm{p}=0.69)$. $\mathrm{LV}$ mass also did not correlate with the extent of LGE $(\mathrm{r}=0.024, \mathrm{p}=$ $0.83)$.

LA volume was significantly larger in HCM patients with dystolic dysfunction than in patients without diastolic

Table 2: LV and LA dimensions and extent of late gadolinium enhancement in HCM patients.

\begin{tabular}{|c|c|c|c|}
\hline & Atrial fibrillation $(n=37)$ & No atrial fibrillation $(n=50)$ & p-value \\
\hline Age (years) & $59 \pm 15$ & $56 \pm 11$ & 0.40 \\
\hline LV ejection fraction (\%) & $56 \pm 10$ & $61 \pm 10$ & 0.07 \\
\hline LV mass $\left(\mathrm{gr} \cdot \mathrm{m}^{-2}\right)$ & $97 \pm 31$ & $99 \pm 34$ & 0.76 \\
\hline LV EDD $(\mathrm{mm})$ & $51 \pm 7$ & $49 \pm 8$ & 0.51 \\
\hline SWT (mm) & $19 \pm 6$ & $18 \pm 4$ & 0.28 \\
\hline PWT (mm) & $10 \pm 3$ & $11 \pm 3$ & 0.28 \\
\hline LVEDV $\left(\mathrm{mL} \cdot \mathrm{m}^{-2}\right)$ & $86 \pm 23$ & $79 \pm 20$ & 0.16 \\
\hline LVESV $\left(\mathrm{mL} \cdot \mathrm{m}^{-2}\right)$ & $37 \pm 14$ & $31 \pm 11$ & 0.07 \\
\hline $\operatorname{LVSV}\left(\mathrm{mL} \cdot \mathrm{m}^{-2}\right)$ & $49 \pm 15$ & $50 \pm 15$ & 0.89 \\
\hline LA volume $\left(\mathrm{mL} \cdot \mathrm{m}^{-2}\right)$ & $68 \pm 24$ & $46 \pm 18$ & 0.0002 \\
\hline LGE (\%) & $12.4 \pm 14.5$ & $6.0 \pm 8.6$ & 0.02 \\
\hline
\end{tabular}

Data are presented as \pm standard deviation. Volumes are indexed to body surface area. EDD $=$ end diastolic dimension, EDV $=$ end diastolic volume, ESV = end systolic volume, LA = left atrial, LGE = late gadolinium enhancement, LV= left ventricular, PWT = posterior wall thickness, SWT $=$ septal wall thickness 
Table 3: Comparison of left ventricular and left atrial dimensions between asymptomatic and symptomatic HCM patients.

\begin{tabular}{llll}
\hline & Asymptomatic HCM (n = 4I) & Symptomatic HCM (n = 46) & p-value \\
\hline Atrial fibrillation & $17(41 \%)$ & $20(43 \%)$ & 0.68 \\
$\quad$ None & $24(59 \%)$ & $26(57 \%)$ & 0.97 \\
$\quad$ Paroxysmal & $15(37 \%)$ & $12(26 \%)$ & 0.41 \\
$\quad$ Persistent & $2(5 \%)$ & $8(17 \%)$ & 0.13 \\
LV ejection fraction $(\%)$ & $60 \pm 10$ & $58 \pm 9.5$ & 0.32 \\
LV mass $\left(\mathrm{gr} \cdot \mathrm{m}^{-2}\right)$ & $99 \pm 30$ & $97 \pm 34$ & 0.84 \\
LV end systolic volume $\left(\mathrm{ml} \cdot \mathrm{m}^{-2}\right)$ & $29 \pm 13$ & $37 \pm 13$ & 0.04 \\
LA volume $\left(\mathrm{ml} \cdot \mathrm{m}^{-2}\right)$ & $48 \pm 21$ & $59 \pm 23$ & 0.03 \\
Extent of LGE $(\%)$ & $6.0 \pm 9.0$ & $11 \pm 13$ & 0.03 \\
\hline
\end{tabular}

Data are presented as \pm standard deviation. Volumes are indexed to body surface area. HCM $=$ hypertrophic cardiomyopathy patients, LA $=$ left atrial. $\mathrm{LGE}=$ late gadolinium enhancement, $\mathrm{LV}=$ left ventricular

dysfunction ( $\mathrm{p}=0.04)$. In HCM patients with diastolic dysfunction the mean extent of LGE tended to be higher compared to HCM patients without diastolic dysfunction. However, this comparison did not reach a significant level (13\% versus $9 \%, \mathrm{p}=0.3$ ).

With multivariate logistic regression analysis, a model using the extent of LGE, LA volume, presence of mitral regurgitation and presence of diastolic dysfunction to predict AF in HCM patients had an r-square of $0.31, \mathrm{p}<0.01$, and revealed that only LA volume and diastolic dysfunction were independently associated with a history of AF in HCM patients $(\mathrm{p}=0.006, \mathrm{p}=0.01$ respectively).
The univariate association between the presence of AF with LA volume and extent of LGE was confirmed with ROC analysis, see figure 1 . The receiver-operator curve indicates that cut-off value for LA volume of $49.8 \mathrm{ml} / \mathrm{m}^{2}$ has optimal discriminative power to predict $\mathrm{AF}$, yielding a sensitivity of $80.8 \%$ and specificity of $64.1 \%$. With respect to the extent of LGE, an optimal cut-off value of 3.3\% was found to have a sensitivity of $76.9 \%$ and a specificity of $59.0 \%$, see figure 1 .

\section{Discussion}

The present CMR study demonstrates that HCM patients with AF had larger LA volumes and displayed more LGE
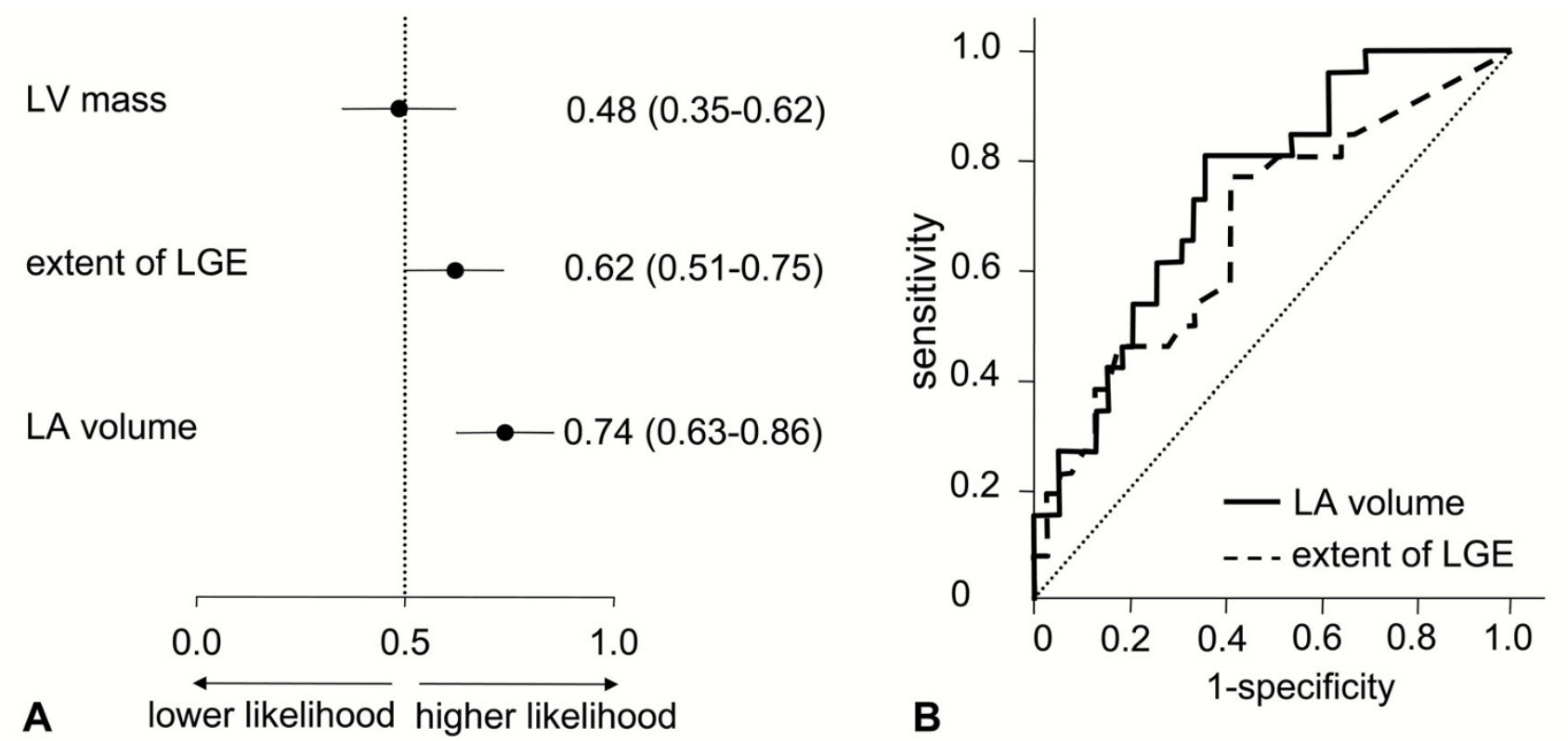

Figure I

Receiver operating characteristic (ROC) curve analysis of left ventricular mass, extent of late gadolinium enhancement (LGE) area and left atrial volume as a predictor of AF. In the panel A, mean area-under-curve with 95\% confidence intervals are presented. In the panel B, ROC curves are presented of extent of LGE and left atrial volume to determine optimal cut-off values. $L A=$ left atrial, $L V=$ left ventricular, $L G E=$ late gadolinium enhancement. 
than HCM patients without AF. LA volume and diastolic dysfunction were independently associated with a history of AF in HCM patients. However, among these two parameters LA volume was the strongest independent determinant of AF. Additionally, LA volume moderately correlated to the extent of LGE irrespective of LV mass. Also, in symptomatic HCM patients in this study, LV endsystolic volumes and LA volumes were larger and extent of LGE was higher compared to asymptomatic HCM patients, which is in line with previous reports $[16,22]$. Interestingly, paroxysmal $\mathrm{AF}$ did not seem to be more prevalent in symptomatic HCM patients than asymptomatic HCM patients, but persistent AF tended to have a higher prevalence in symptomatic HCM patients compared to asymptomatic HCM patients.

$\mathrm{AF}$ is a commonly reported complication of HCM $[2,4,8,10]$. Established AF is uncommon in the young, but in adults its prevalence can reach up to $30 \%$ [23]. Remarkably, the prevalence of AF in our study population was higher than previously reported. This may in part be due to the large number of follow-up visits, ECGs and 24 hours ambulatory Holter ECG recordings in the majority of patients. Since AF is considered a key determinant of HCM-related morbidity and mortality [7,10-12], the identification of predictors of $\mathrm{AF}$ is of paramount clinical importance.

In a community-based HCM population, Olivotto et al. found that the strongest predictor of AF was increased LA volume, independent of age and functional NYHA functional class [11]. In addition to LA volume, Losi et al. reported that LA fractional shortening and age were independent predictors for the development of AF in HCM patients [23]. However, interpretation of these studies may be difficult, while LA volume measurement may be hindered by a poor acoustic window which is inherent to echocardiography. By using CMR, we merely omitted these limitations to measure LA volume, and confirmed the importance of LA volume in relation to AF in HCM patients. In this CMR study, a LA volume cut-off value of $50 \mathrm{ml} \cdot \mathrm{m}^{-2}$ had an optimal discriminative power to predict $\mathrm{AF}$, yielding a sensitivity of $81 \%$ and a specificity of $64 \%$. This cut-off value is comparable with the results from Losi et al, who found a cut-off value of $44 \mathrm{ml} \cdot \mathrm{m}^{-2}$ determined with echocardiography [23]. The strong relation between LA dilation and AF in HCM patients may be explained by the electrical and structural remodeling that occur in the process of LA dilation, including shortening of the atrial effective refractory period and local conduction delay [24].

In addition, AF was associated with LGE in HCM patients. However, not the presence but the extent was shown to be indicative for $\mathrm{AF}$. This is in line with the findings of a necropsy study of HCM patients performed by Yamaji et al [25], who found that the extent of LV fibrosis was significantly higher in HCM patients with $\mathrm{AF}(24.4 \pm 0.6 \%)$ than without AF $(17.6 \pm 0.7 \%)$. The total fibrotic burden in the LV found by Yamaji et al was higher compared to our findings. This difference might be explained by the fact that patients in this necropsy study died from progressive $\mathrm{HCM}$, which has been associated with an increased fibrotic burden in comparison with milder forms of HCM [16]. Moreover, systematic underestimation of fibrosis quantification is an important limitation of LGE imaging, by which only focal, but not diffuse areas of fibrosis can be visualized and quantified. In addition, the LV must remain in diastasis for at least $100 \mathrm{~ms}$ for optimal quality of the LGE images. Therefore, in this study, only HCM patients with $\mathrm{AF}$ who had acceptable rate control were referred for CMR, which may have introduced a selection bias into our analysis.

The underlying mechanisms relating LV LGE with AF are unknown. The histological background of LGE in HCM patients has been suggested to be myocardial scarring and/or interstitial fibrosis [15]. Focal areas of myocardial scarring have been related to ventricular arrhythmias, but may also serve as anatomical substrates for AF $[17,18]$. Interstitial fibrosis is produced by fibroblasts that are activated by cardiotrophic mediators, such as angiotensin II. Interstitial fibrosis together with impaired relaxation of the cardiomyocyte through altered $\mathrm{Ca}^{2+}$-handling may ultimately lead to diastolic dysfunction of the LV and subsequent LA dilation and explain the relation between the extent of LGE, LA dilation and subsequent $\operatorname{AF}[26,27]$. This statement was supported by the moderate, but significant correlation between LGE and LA volume found in this study. Additionally, patients with diastolic dysfunction revealed considerably more LGE than patients without, indicating a potential relationship between LGE and diastolic function.

In contrast to patients with hypertension and in normal population $[28,29]$, LV mass did not correlate to LA dilation in these HCM patients, see figure 2. In HCM patients, mitral regurgitation, originating from systolic anterior motion of the mitral valve leaflet due to LV outflow tract obstruction and increased interstitial fibrosis and myocardial scarring also importantly attribute to increased LA volume. However, no quantitative data evaluating the relative contribution of the extent of LGE, LV diastolic dysfunction and mitral regurgitation to LA dilation in HCM patients are yet available.

Mitral regurgitation is very common in patients with HCM. Also, in our study population mitral regurgitation was present in 39 (45\%) patients. However, mitral regurgitation was not a independent predictor of AF in HCM patients. Similar to our results, Losi et al. found that mitral 

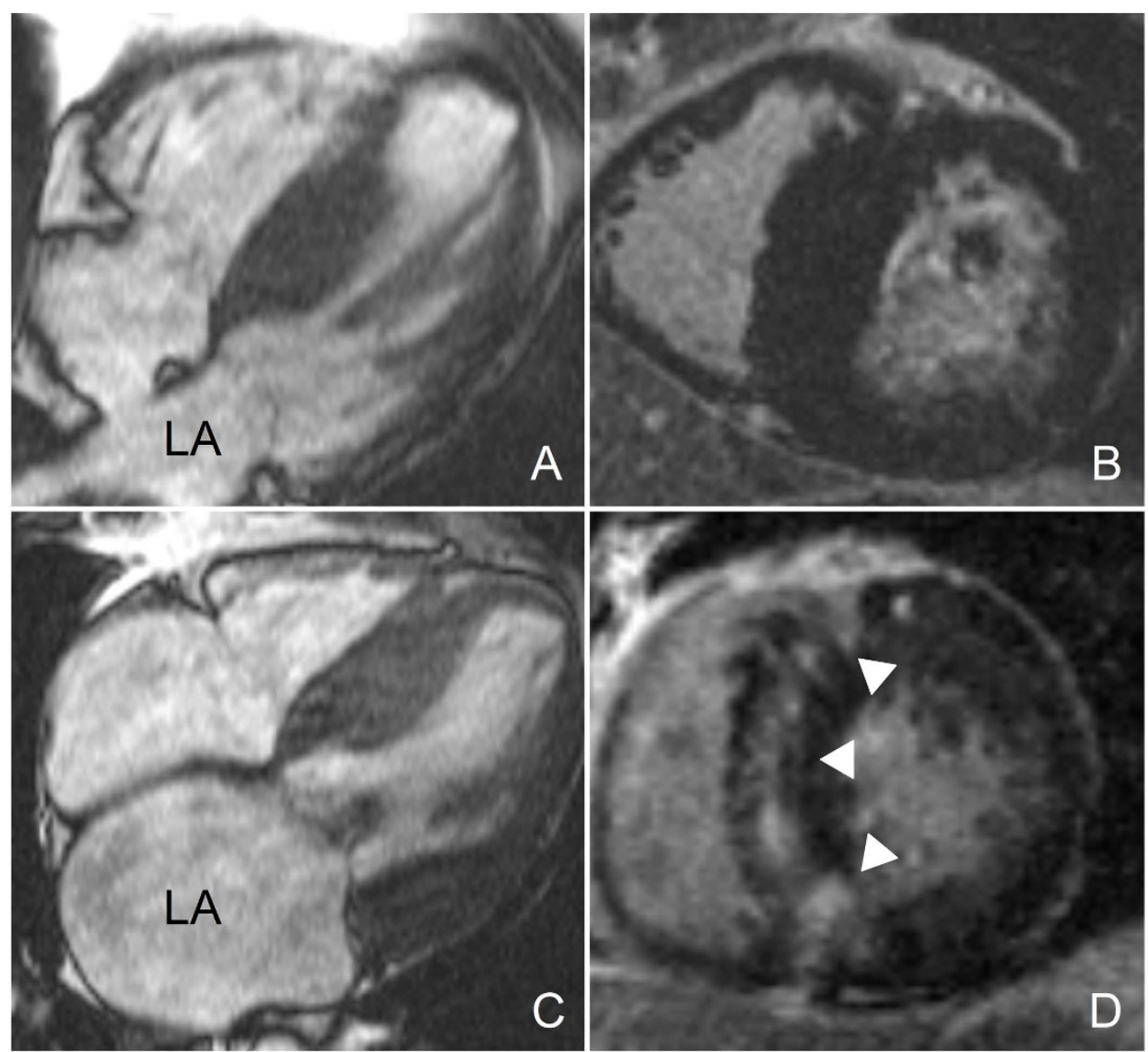

Figure 2

HCM patients with comparable left ventricular mass and different left atrial size. A, C. End diastolic 4 chamber cine images. Note that the left atrium of the first patient $(A)$ is smaller compared to the left atrium of the second patient (C), despite comparable LV mass. The end diastolic short axis LGE images (B, D) reveal that the first patient (B) has no LGE, while the second patient (D) has extensive LGE of the septum, as indicated by the white arrowheads.

regurgitaiton was not a determinant of AF in any model of multivariate analysis [23].

\section{Clinical implication}

Should further studies confirm the pathogenic role of fibrosis in diastolic dysfunction and subsequent LA dilation in HCM, different treatment options aimed at stopping or even reversing the process of fibrosis could gain clinical importance. For example, angiotensin II blockade, statins and aldosteron antagonists, have already been demonstrated experimentally to cause regression of fibrosis, hypertrophy and disarray in animal HCM models [3032]. Moreover, the strong relation between LA dilation and AF may justify to use LA size $>50 \mathrm{~mL} \cdot \mathrm{m}^{-2}$ determined with CMR as an indication to aggressively search for the presence of AF.

\section{Limitations}

For cine and LGE imaging, we used a gap of 5 and $4 \mathrm{~mm}$ between slices for full coverage of the left ventricle, thereby omitting 40 to $50 \%$ of total myocardium. This may potentially have introduced inaccuracy of volume and mass measurement; however, in a comparative study performed by Hogan et al, contiguous acquisition of slices and acquisition with $4 \mathrm{~mm}$ showed comparable accuracy and yielded comparable volumes [33]. As mentioned previously, LGE imaging systemically underestimates total burden of diffuse fibrosis, but a recent CMR study has shown promising results to overcome this limitation [34]. In addition, the temporal resolution of CMR cine imaging was not sufficient to adequately assessed LV diastolic dysfunction. Therefore we used the echocardiographic data to evaluate the diastolic function.

In conclusion, HCM patients with a history of AF display significantly more LGE than HCM patients without AF. However, the extent of LGE is inferior to the LA size for predicting AF prevalence. LA dilation is the strongest determinant of AF in HCM patients, and is related to the extent of LGE in the LV, irrespective of LV mass. 


\section{Abbreviations}

CMR: cardiovascular magnetic resonance; AF: atrial fibrillation; LGE: late gadolinium enhancement; HCM: hypertrophic cardiomyopathy; EF: ejection fraction; LV: left ventricular; LA: left atrium; trueFISP: Balanced segmented steady state free precession.

\section{Competing interests}

The authors declare that they have no competing interests.

\section{Authors' contributions}

TP performed and evaluated CMR images, participated in study-design, figures and tables and drafted the manuscript. TG contributed equally to the writing of this manuscript. SF participated in study-design and evaluation of CMR data. CD participated in the revision of the manuscript and the evaluation of the echocardiograpical data. AS participated in study design and its coordination. DH participated in collection and evaluation of clinical data. TS participated in study-design and revision of the manuscript. CW participated in study-design and statistical analysis. DD participated in study-design and evaluation of CMR-images. SS participated in study-design and evaluation of CMR-images. AvR was responsible for the revision of the manuscript. MB Participated in study-design, scientific and clinical advice concerning HCM and was responsible for the revision of the manuscript. All authors read and approved the final manuscript.

\section{Acknowledgements}

T.G. is supported by a grant from the Netherlands Heart Foundation, grant number 2006B2I3.

\section{References}

I. Maron BJ: Hypertrophic cardiomyopathy: a systematic review. JAMA 2002, 287:1308-20.

2. Cecchi F, Montereggi A, Olivotto I, Marconi P, Dolara A, Maron B]: Risk for atrial fibrillation in patients with hypertrophic cardiomyopathy assessed by signal averaged $\mathbf{P}$ wave duration. Heart 1997, 78:44-9.

3. Maron BJ: Hypertrophic cardiomyopathy. Lancet 1997, 350:127-33.

4. Olivotto I, Maron BJ, Cecchi F: Clinical significance of atrial fibrillation in hypertrophic cardiomyopathy. Curr Cardiol Rep 200I, 3:|4|-6.

5. Spirito P, Seidman CE, McKenna WJ, Maron BJ: The management of hypertrophic cardiomyopathy. N Engl J Med 1997, 336:775-85.

6. Furlan AJ, Craciun AR, Raju NR, Hart N: Cerebrovascular complications associated with idiopathic hypertrophic subaortic stenosis. Stroke 1984, 1 5:282-4.

7. Glancy DL, O'Brien KP, Gold HK, Epstein SE: Atrial fibrillation in patients with idiopathic hypertrophic subaortic stenosis. $\mathrm{Br}$ Heart J 1970, 32:652-9.

8. Higashikawa M, Nakamura Y, Yoshida M, Kinoshita M: Incidence of ischemic strokes in hypertrophic cardiomyopathy is markedly increased if complicated by atrial fibrillation. Jpn Circ J 1997, 61:673-8I.

9. Maron BJ, Casey SA, Poliac LC, Gohman TE, Almquist AK, Aeppli DM: Clinical course of hypertrophic cardiomyopathy in a regional United States cohort. JAMA 1999, 281:650-5.

10. Stafford WJ, Trohman RG, Bilsker M, Zaman L, Castellanos A, Myerburg RJ: Cardiac arrest in an adolescent with atrial fibrillation and hypertrophic cardiomyopathy. I Am Coll Cardiol 1986, 7:701-4.

II. Olivotto I, Cecchi F, Casey SA, Dolara A, Traverse JH, Maron BJ: Impact of atrial fibrillation on the clinical course of hypertrophic cardiomyopathy. Circulation 200I, 104:2517-24.

12. Bottini PB, Carr AA, Prisant LM, Flickinger FW, Allison JD, Gottdiener JS: Magnetic resonance imaging compared to echocardiography to assess left ventricular mass in the hypertensive patient. Am J Hypertens 1995, 8:22I-8.

13. Lorenz CH, Walker ES, Morgan VL, Klein SS, Graham TP Jr: Normal human right and left ventricular mass, systolic function, and gender differences by cine magnetic resonance imaging. J Cardiovasc Magn Reson 1999, I:7-21.

14. Choudhury L, Mahrholdt H, Wagner A, et al:: Myocardial scarring in asymptomatic or mildly symptomatic patients with hypertrophic cardiomyopathy. J Am Coll Cardiol 2002, 40:2 I 56-64.

15. Moon JC, Reed E, Sheppard MN, et al.: The histologic basis of late gadolinium enhancement cardiovascular magnetic resonance in hypertrophic cardiomyopathy. J Am Coll Cardiol 2004, 43:2260-4.

16. Moon JC, McKenna WJ, McCrohon JA, Elliott PM, Smith GC, Pennell DJ: Toward clinical risk assessment in hypertrophic cardiomyopathy with gadolinium cardiovascular magnetic resonance. J Am Coll Cardiol 2003, 4I:I56I-7.

17. Teraoka K, Hirano M, Ookubo $H$, et al: Delayed contrast enhancement of MRI in hypertrophic cardiomyopathy. Magn Reson Imaging 2004, 22: I55-6|.

18. Adabag AS, Maron BJ, Appelbaum E, Harrigan CJ, Buros JL, Gibson CM, Lesser JR, Hanna CA, Udelson JE, Manning WJ, Maron MS: Occurrence and frequency of arrhythmias in hypertrophic cardiomyopathy in relation to delayed enhancement on cardiovascular magnetic resonance. J Am Coll Cardiol 2008, $5 \mathrm{I}: 1369-74$

19. Maron BJ, Towbin JA, Thiene G, Antzelevitch C, Corrado D, Arnett D, Moss AJ, Seidman CE, Young JB: Contemporary definitions and classification of the cardiomyopathies: an American Heart Association Scientific Statement from the Council on Clinical Cardiology, Heart Failure and Transplantation Committee; Quality of Care and Outcomes Research and Functional Genomics and Translational Biology Interdisciplinary Working Groups; and Council on Epidemiology and Prevention. Circulation 2006, I 1 3:1807-16.

20. Simonetti OP, Kim RJ, Fieno DS, Hillenbrand HB, Wu E, Bundy JM, Finn JP, Judd RM: An improved MR imaging technique for the visualization of myocardial infarction. Radiology 200I, 218:215-23.

21. Sievers B, Kirchberg S, Addo M, Bakan A, Brandts B, Trappe HJ: Assessment of left atrial volumes in sinus rhythm and atrial fibrillation using the biplane area-length method and cardiovascular magnetic resonance imaging with TrueFISP. J Cardiovasc Magn Reson 2004, 6:855-63.

22. Sachdev V, Shizukuda Y, Brenneman CL, Birdsall C, Waclawiw M, Arai A, Mohiddin S, Tripodi D, Fananapazir L, Plehn J: Left atrial volumetric remodeling is predictive of functional capacity in nonobstructive hypertrophic cardiomyopathy. Am Heart J 2005, 149:730-6.

23. Losi MA, Betocchi S, Aversa M, Lombardi R, Miranda M, D'Alessandro G, Cacace A, Tocchetti C, Barbati G, Chiariello M: Determinants of atrial fibrillation development in patients with hypertrophic cardiomyopathy. Am J Cardiol 2004, 94:895-900.

24. Liu T, Li GP: Potential mechanisms between atrial dilatation and atrial fibrillation. Am Heart J 2006, I $5 \mathrm{I}: \mathrm{el}$.

25. Yamaji K, Fujimoto S, Yutani C, Ikeda Y, Mizuno R, Hashimoto T, Nakamura S: Does the progression of myocardial fibrosis lead to atrial fibrillation in patients with hypertrophic cardiomyopathy? Cardiovasc Pathol 200I, 10:297-303.

26. Tsoutsman T, Lam L, Semsarian C: Genes, calcium and modifying factors in hypertrophic cardiomyopathy. Clin Exp Pharmacol Physiol 2006, 33:139-45.

27. Papavassiliu T, Schnabel P, Schroder M, Borggrefe M: CMR scarring in a patient with hypertrophic cardiomyopathy correlates well with histological findings of fibrosis. Eur Heart J 2005, 26:2395.

28. Germans T, Gotte MJ, Nijveldt R, Spreeuwenberg M, Beek A, Bronzwaer J, Visser C, Paulus W, van Rossum AC: Effects of aging on left atrioventricular coupling and left ventricular filling 
assessed using cardiac magnetic resonance imaging in healthy subjects. Am J Cardiol 2007, I00: I22-7.

29. Gottdiener JS, Reda DJ, Williams DW, Materson BJ: Left atrial size in hypertensive men: influence of obesity, race and age. Department of Veterans Affairs Cooperative Study Group on Antihypertensive Agents. J Am Coll Cardiol I 997, 29:65I-8.

30. Lim DS, Lutucuta S, Bachireddy P, et al.: Angiotensin II blockade reverses myocardial fibrosis in a transgenic mouse model of human hypertrophic cardiomyopathy. Circulation 200I, 103:789-9|.

31. Patel R, Lim DS, Reddy D, et al:: Variants of trophic factors and expression of cardiac hypertrophy in patients with hypertrophic cardiomyopathy. J Mol Cell Cardiol 2000, 32:2369-77.

32. Tsybouleva N, Zhang L, Chen S, et al.: Aldosterone, through novel signaling proteins, is a fundamental molecular bridge between the genetic defect and the cardiac phenotype of hypertrophic cardiomyopathy. Circulation 2004, 109:1284-9|

33. Maureen C, Hogan MC, Petersen SE, Hudsmith LE, Francis JM, Neubauer S, Robson MD: Effects of steady state free precession parameters on cardiac mass, function, and volumes. Int J Cardiovasc Imaging 2007, 23:583-9.

34. Kehr E, Sono M, Chugh SS, Jerosch-Herold M: Gadoliniumenhanced magnetic resonance imaging for detection and quantification of fibrosis in human myocardium in vitro. Int J Cardiovasc Imaging 2008, 24:6I-8.

Publish with Bio Med Central and every scientist can read your work free of charge

"BioMed Central will be the most significant development for disseminating the results of biomedical research in our lifetime. "

Sir Paul Nurse, Cancer Research UK

Your research papers will be:

- available free of charge to the entire biomedical community

- peer reviewed and published immediately upon acceptance

- cited in PubMed and archived on PubMed Central

- yours - you keep the copyright 